University of Nebraska - Lincoln

DigitalCommons@University of Nebraska - Lincoln

\title{
To be or not to be a locust? A comparative analysis of behavioral phase change in nymphs of Schistocerca americana and S. gregaria
}

\author{
Gregory A. Sword \\ United States Department of Agriculture
}

Follow this and additional works at: https://digitalcommons.unl.edu/usdaarsfacpub

Part of the Agricultural Science Commons

Sword, Gregory A., "To be or not to be a locust? A comparative analysis of behavioral phase change in nymphs of Schistocerca americana and S. gregaria" (2003). Publications from USDA-ARS / UNL Faculty. 381.

https://digitalcommons.unl.edu/usdaarsfacpub/381

This Article is brought to you for free and open access by the U.S. Department of Agriculture: Agricultural Research Service, Lincoln, Nebraska at DigitalCommons@University of Nebraska - Lincoln. It has been accepted for inclusion in Publications from USDA-ARS / UNL Faculty by an authorized administrator of DigitalCommons@University of Nebraska - Lincoln. 


\title{
To be or not to be a locust? A comparative analysis of behavioral phase change in nymphs of Schistocerca americana and $S$. gregaria
}

\author{
Gregory A. Sword * \\ United States Department of Agriculture, Agricultural Research Service, 1500 N. Central Avenue, Sidney, MT 59270, USA
}

Received 4 December 2002; received in revised form 28 March 2003; accepted 2 April 2003

\begin{abstract}
Phenotypic plasticity in behavior induced by high rearing density is often part of a migratory syndrome in insects called phase polyphenism. Among locust species, swarming and the expression of phase polyphenism are highly correlated. The american grasshopper, Schistocerca americana, rarely swarms even though it is closely related to the swarming Old World desert locust, $S$. gregaria, as well as two swarming New World locusts. Anecdotal field observations of locust-like behavior in S. americana indicate that it may express behavioral phase polyphenism, but empirical investigations are lacking. In this study, I tested the hypothesis that $S$. americana expresses locust-like density-dependent changes in behavior during both the first and final nymphal instars. I then compared the expression of behavioral phase change between $S$. americana and $S$. gregaria. First instar S. americana exhibited significant geographic variation in behavior with grasshoppers from a North Carolina population expressing more pronounced density-dependent changes relative to grasshoppers from a Texas population. The behavior of final instar S. americana was only slightly affected by rearing density and there was no evidence for a difference between populations. Comparison with $S$. gregaria revealed that the magnitude of density-dependent behavioral change, particularly among final instar nymphs, was much reduced in S. americana.
\end{abstract}

Published by Elsevier Science Ltd.

Keywords: Phenotypic plasticity; Density-dependent; Behavior; Phase polyphenism

\section{Introduction}

Many insects exhibit phenotypic plasticity in response to changes in their rearing density. Increases in population density can elicit physiological responses that manifest themselves as phenotypic changes in behavior, color, metabolism, development, and morphology (reviewed in Pener, 1991; Pener and Yerushalmi, 1998; Applebaum and Heifetz, 1999; Simpson et al., 1999). Density-dependent phenotypic plasticity was initially identified in locusts (Uvarov, 1921) and termed phase polymorphism (Uvarov, 1966). The term phase polyphenism is actually a more appropriate descriptor of the phenomenon, because it implies environmentally determined phenotypic changes as opposed to genetic polymorphism (Dingle, 1996). Phase polyphenism

\footnotetext{
* Tel.: +1-406-433-9429; fax: +1-406-433-5038.

E-mail address: gsword@sidney.ars.usda.gov (G.A. Sword).
}

appears to be an adaptation to the stresses imposed by intraspecific competition at high population density and tends to produce individuals that migrate en mass, presumably to escape the deteriorating conditions within their crowded local habitat (Fescemyer, 1993; Dingle, 1996). Although density-dependent phase polyphenism is expressed to varying degrees among members of the Coleoptera, Lepidoptera, Hemiptera, and Homoptera, it is most commonly associated with locusts in the Orthoptera (Applebaum and Heifetz, 1999).

Among locusts, behavior is one of the first characters to vary in response to changes in rearing density. Locust behavioral phase transition is typified by changes in activity level and mutual attraction among individuals. "Solitarious" phase phenotypes are produced under isolated rearing conditions and "gregarious" phase phenotypes are produced under crowded conditions. Studies of the desert locust, Schistocerca gregaria (Forskål), have shown that solitarious phase locusts tend to be inactive and avoid each other, while gregarious phase individuals 
are more active and typically orient toward one another (Simpson et al., 1999 and citations therein). The simultaneous expression of mutual attraction and increased activity presumably contributes to the formation of large mobile hopper bands and adult swarms that are characteristic of locust species under outbreak conditions (Uvarov, 1977). Increases in local population density are initially facilitated by favorable abiotic conditions such as weather. As local densities increase, so does the probability of contact among individuals, especially where resources are clumped at small spatial scales (Bouaïchi et al., 1996; Collett et al., 1998; Despland et al., 2000; Despland and Simpson 2000a, b). Increased contact among individuals utilizing these resources is significant because direct tactile stimulation mediates behavioral phase change (Simpson et al., 2001). Once in the gregarious behavioral phase, locusts may then undergo phase changes in other traits. Some of these changes, such as the expression of density-dependent prophylaxis and density-dependent warning coloration, may act as positive feedbacks on population growth and further contribute to the outbreak process (Sword et al., 2000; Sword and Simpson, 2000; Wilson et al., 2002).

The ability to express extreme phase polyphenism is common among the swarming Schistocerca locusts. However, not all members of the genus exhibit pronounced density-dependent phase changes (Kevan, 1943; Rowell and Cannis, 1971; Harvey, 1981). Thus, comparative analyses among closely related Schistocerca species offer potentially informative systems for studying the physiological mechanisms underlying phase polyphenism in locusts as well as the ecology and evolution of phase polyphenism in general (e.g. Sword, 2002).

The american grasshopper, S. americana (Drury), is widespread throughout the southeastern United States. This species is of occasional agricultural importance and can be a devastating Citrus pest in Florida (Capinera, 1993). S. americana rarely swarms despite the fact that it is closely related to three other swarming Schistocerca locusts. It is reproductively isolated from the Old World desert locust, $S$. gregaria, but not from the two most important swarming locusts in the New World, S. piceifrons (Walker), the Central American locust, and S. cancellata (Serville), the South American locust (Harvey, 1979, 1982; Jago et al., 1979). Swarms of the Central American locust occur as far north as the Tropic of Cancer (Harvey, 1983) and its range overlaps with that of S. americana in northeastern Mexico (Harvey, 1981) presenting the potential for gene flow between the two species. S. americana exhibits a density-dependent nymphal color change similar to the swarming Schistocerca locusts (Harvey, 1981) and has been reported to express locust-like behaviors in the field. $S$. americana has been observed to form dense nymphal aggregations, move in unison as juveniles, and fly in swarms as adults (Kuitert and Connin, 1952; Capinera et al., 2001). Importantly, quantitative analyses of density-dependent behavioral phase change in S. americana are lacking (Harvey, 1981). In this study, I examine the expression of densitydependent behavioral phase polyphenism in juvenile $S$. americana and compare its expression to that of the desert locust, $S$. gregaria.

\section{Methods}

\subsection{Rearing}

Pre-reproductive $S$. americana adults were collected in two low-density North American populations separated by approx. $1870 \mathrm{~km}$ during September and October of 2001. North Carolina (NC) insects were collected in fields near Schenk forest outside of Raleigh in Wake County, NC. Texas (TX) insects were collected in fields along the shore of Lake Georgetown in Williamson County, TX. Grasshoppers were returned to the laboratory for rearing and observation. Individual mating pairs were established in 81 clear plastic tubs with wire mesh lids. Cages were wrapped in white paper to visually isolate them from each other and maintained in an environment chamber at $30{ }^{\circ} \mathrm{C}, 14: 10 \mathrm{~L}: \mathrm{D}$. Insects were daily fed fresh romaine lettuce, seedling wheat and wheat bran. Egg pods were obtained in 110x43 mm diameter plastic tubes filled with moist sand (10 parts sand:1 part water). Following oviposition, tubes were sealed with a plastic lid, incubated at $30{ }^{\circ} \mathrm{C}$ and monitored for hatching which typically occurred in $21 \mathrm{~d}$. Pods were not split prior to hatching.

Upon hatching, six neonates per pod from six mating pairs per population were used to establish isolationreared treatment groups for each population. This distributed potential family level variation in a manner similar to that employed in rearing isolated $S$. gregaria (see Roessingh et al., 1993, as well as below). Isolated insects were individually reared in $473 \mathrm{~cm}^{3}$ cylindrical clear plastic cages with wire mesh tops wrapped in white paper for visual isolation. Isolated cages were housed in an environment chamber $(184 \times 184 \times 78 \mathrm{~cm})$ at $30{ }^{\circ} \mathrm{C}$, 14:10 L:D with one complete air exchange every 3.5 min to minimize olfactory contact among the isolated individuals due to the potential for olfactory cues to affect nymphal phase behavior (Heiftez et al., 1996; Despland, 2001). Crowded first instar treatments were established by placing hatchlings in groups of 40-50 in 61 clear plastic buckets with wire mesh lids wrapped in white paper. Following the first instar behavioral observations, all crowded groups within each population were combined in larger $33.5 \times 35 \times 47 \mathrm{~cm}$ screen stock cages and reared under crowded conditions (approx. 300 insects per population) through the final instar. Crowded cages were maintained apart from the isolated insects in 
a different environment chamber at $30{ }^{\circ} \mathrm{C}, 14: 10 \mathrm{~L}: \mathrm{D}$. All insects were daily fed fresh Romaine lettuce, seedling wheat, and wheat bran. Grasshoppers in the isolated and crowded treatments developed the expected lowand high-density nymphal color phenotypes (green vs. yellow-and-black or orange-and-black, respectively) (Harvey, 1981), indicating that the rearing densities used here were sufficient to induce density-dependent phenotypic changes.

The isolated (solitarious phase) and crowded (gregarious phase) S. gregaria nymphs used here were part of the breeding stock colony maintained in the Department of Zoology, University of Oxford. Isolated and crowded rearing conditions as well as the breeding regime are described in Roessingh et al. (1993) and Simpson et al. (1999). The solitarious phase locusts I observed had been reared in isolation for two generations. The gregarious phase locusts had been reared under crowded conditions for many generations.

Although both $S$. americana and $S$. gregaria were reared under isolated and crowed conditions for this study, they were not reared under completely identical conditions. There were slight differences in cage size, feeding regime, photoperiod and olfactory isolation parameters (see Roessingh et al. (1993) for detailed S. gregaria protocol). Although I assumed the behavioral effects of these uncontrolled variables to be negligible, the possibility of an effect cannot be eliminated. Thus, the interspecific comparisons made in this study should be regarded as coarse comparisons.

\subsection{Behavioral assay}

I employed an individual-based behavioral assay developed for the analysis of phase-related behaviors in S. gregaria. See Roessingh et al. (1993) and Simpson et al. (1999) for detailed descriptions of the assay, observation arena, and examples of their use in locust behavioral studies. S. americana and S. gregaria observations were conducted at the USDA Northern Plains Agricultural Research Laboratory and University of Oxford, respectively. To facilitate direct comparison of the different species' behavior, I observed the behavior of first and final instar nymphs of both species in observational arenas constructed to the same specifications. The laboratory conditions during the observations were as similar as possible with the lighting, temperature and stimulus group sizes (described below) being identical for both species.

First instar (2-3 d post-hatch) behaviors were assayed in a rectangular, white Perspex arena measuring $35.5 \times 15 \mathrm{~cm}$ and $10 \mathrm{~cm}$ high (see Islam et al., 1994a, b for description and applications of the first instar arena). One of two stimulus chambers separated from the main observation area by perforated clear Perspex panels at both ends of the arena contained 10 first instar crowd- reared insects. A Cartesian grid was plotted on the floor of the arena to allow the position of the experimental insect during the assay to be recorded. Immediately prior to observation, the experimental insects were placed inside a modified blackened $10 \mathrm{ml}$ syringe, allowed to settle for $5 \mathrm{~min}$, and gently introduced into the arena through a hole in center of the floor. Their behaviors in response to the stimulus group were observed for $5 \mathrm{~min}$ and recorded using a laptop computer programmed as an event recorder. The observations of final-instar nymphs (2-5 d post-ecdysis) were conducted in an identical manner using a scaled-up arena measuring 57x30 $\mathrm{cm}$ and $10 \mathrm{~cm}$ high with 10 crowd-reared final instars as a stimulus group and a $20 \mathrm{ml}$ blackened syringe as a tube and plunger (see Roessingh et al., 1993). All observations were conducted at $30{ }^{\circ} \mathrm{C}$. Thirty $\mathrm{S}$. americana nymphs per isolated and crowded rearing treatment (approx. 6 per family) from each of the TX and NC populations were observed in the arena during the first and final instars. Sixty isolated and 60 crowd-reared $S$. gregaria nymphs per instar were similarly observed.

\subsection{Analysis}

I selected six activity-related and two attractionrelated behaviors for analysis. Based on previous analyses of locust behavior, values for the activity-related variables are predicted to increase among crowded locusts. Similarly, values for the attraction-related variables should indicate that crowd-reared locusts are more likely to orient toward other locusts (Roessingh et al., 1993; Simpson et al., 1999). The six activity-related (ivi) and two attraction-related variables (vii and viii) were: (i) Climb time, proportion of time spent climbing the stimulus chamber walls during the assay; (ii) turn angle, average turn angle; (iii) turn frequency, number of turns divided by assay duration; (iv) walk frequency, the number of walking events divided by assay duration; (v) walk speed, total walk distance divided by total walking time; (vi) walk time, proportion of time spent walking during the assay; (vii) end position, the position of the insect relative to the stimulus group along the $X$-axis of the arena at the end of the assay; and (viii) overall position, the amount of time spent in the third of the arena closest to the stimulus group minus the amount of time spent in the third of the arena furthest from the stimulus group, divided by assay duration.

To determine the overall effects of population of origin and rearing density on $S$. americana behavior, I employed a 2x2 factorial non-parametric MANOVA (NPMANOVA)(Anderson, 2000) with POPULATION and DENSITY as fixed factors. NPMANOVA uses a test statistic analogous to Fisher's $F$-ratio that is calculated from a distance matrix generated from the original data. $P$-values are obtained using permutations. The advantages of this permutation-based test are that it requires 
no assumptions regarding the number, distributions, or correlations among variables, and that it can partition factor variation in any two-way design (Anderson, 2001). Behavioral variables were rank-normal transformed in SPSS 10.0 (SPSS Inc., 1999) prior to analysis in NPMANOVA to standardize for differences in scale (Anderson, 2001). Analysis was based on a dissimilarity matrix of Euclidian distances among all the variables using the permutation of residuals option for 9999 iterations. The effects of rearing density on the individual behavioral variables were further explored within each population using posthoc non-parametric Mann-Whitney $U$-tests corrected for ties in StatView 5.0.1 (SAS Institute, 1998). A sequential Dunn-Sidák method Bonferroni correction with an experimentwise error rate of $\alpha=0.05$ was applied to control for multiple comparisons (Sokal and Rolf, 1995). For the eight univariate comparisons, the Dunn-Sidák method provided eight associated $\alpha$ values against which the ranked $P$ values from the Mann-Whitney tests were sequentially compared to determine significance.

I also used NPMANOVA as described above to compare the overall behavioral effects of rearing density between $S$. americana and $S$. gregaria using SPECIES and DENSITY as fixed factors. Due to a significant POPULATION $\times$ DENSITY interaction detected within first instar S. americana (see Section 3), I compared only the more extreme behavioral response of the first instar $S$. americana from NC to the behavior of $S$. gregaria. To satisfy the balanced sample size requirement of NPMANOVA, I randomly sub-sampled the first instar $S$. gregaria data within each density treatment to create a balanced data set of $N=120$ individual observations. This provided 30 individuals per factor level $($ SPECIES $\times$ DENSITY) corresponding to the smallest treatment groups of $N=30$ isolated and crowded $S$. americana from NC. The entire first instar $S$. gregaria data set of $N=60$ per density treatment was used in the follow-up univariate comparisons. No significant variation was detected between the $S$. americana populations in the final instar and I carried out both the NPMANOVA as well as the follow-up univariate comparisons using the data from both populations combined. This yielded a total of $N=240$ observations with $N=$ 60 insects per each SPECIES $\times$ DENSITY factor level.

\section{Results}

\subsection{S. americana behavior}

S. americana nymphs exhibited density-dependent behavioral changes, but their behavioral responses to isolated and crowded rearing conditions varied between populations as well as across developmental stages.

\subsubsection{First instars}

Grasshoppers from the TX and NC populations did not behave in the same manner in response to isolated and crowded rearing conditions. The overall behavioral difference between populations is evidenced by the significant POPULATION $\times$ DENSITY interaction term detected in NPMANOVA (Table 1). Follow-up univariate comparisons of the six activity-related and two attraction-related behaviors between different density treatments within each population indicated that the difference between populations was primarily due to increased activity at high population density among the NC insects, but not the TX insects. Following correction for multiple comparisons, $\mathrm{NC}$ insects exhibited significant density-dependent increases in three activity-related behaviors, Walk frequency $(U=381.0 ; P<0.0001$; associated $\alpha=0.0064)$, Walk speed $(U=252.5 ; P=$ 0.0008 ; associated $\alpha=0.0085$ ), and Walk time ( $U=$ 225.0; $P=0.0001$; associated $\alpha=0.0073$ ) (Fig. 1d-f). No significant differences were detected between the isolated and crowded first instars from TX (Fig. 1).

\subsubsection{Final instars}

Unlike first instar behavior, the overall behavioral response of final instar $S$. americana to changes in rearing density did not differ between the TX and NC populations. Rearing density did have an overall effect on final instar S. americana behavior, but their behavioral change in response to density was independent of their population of origin. This is reflected in the significant effect of DENSITY, but not POPULATION or the interaction term detected in NPMANOVA (Table 1). Followup univariate comparisons revealed that in contrast to the multiple significant density-dependent changes observed among the first instar insects (Fig. 1), only Climb time increased significantly among crowded final instar insects $(U=1250.0 ; P=0.0032 ;$ associated $\alpha=$ 0.0064)(Fig. 2a).

\subsection{Interspecific comparisons}

S. americana and S. gregaria differed in their patterns of expression of behavioral phase polyphenism as both first and final instars.

\subsubsection{First instars}

Among the first instars, the multivariate comparison of behaviors yielded a significant SPECIES $\times$ DENSITY interaction indicating that $S$. americana and $S$. gregaria responded in different ways to isolated and crowded rearing densities (Table 2). Follow-up univariate comparisons revealed that the difference between first instars was largely due to significant density-dependent increases among $S$. gregaria, but not $S$. americana, in Climb time $(U=937.0 ; P<0.0001$; associated $\alpha$ $=0.0085)$ and Turn frequency $(U=675.0 ; P<$ 
Table 1

Non-parametric MANOVA of the effects of population of origin (POPULATION) and rearing density (DENSITY) on the overall behavior of first and final instar $S$. americana from TX and NC

\begin{tabular}{|c|c|c|c|c|c|c|}
\hline Instar & Source & $d f$ & SS & MS & $F$ & $P$ \\
\hline \multirow[t]{4}{*}{ First } & POPULATION & 1 & 15.4177 & 15.4177 & 2.3403 & 0.056 \\
\hline & DENSITY & 1 & 20.3791 & 20.3791 & 3.0933 & 0.017 \\
\hline & POPULATION $\times$ DENSITY & 1 & 21.6164 & 21.6164 & 3.2812 & 0.011 \\
\hline & Residual & 116 & 764.2134 & 6.5880 & & \\
\hline \multirow[t]{4}{*}{ Final } & POPULATION & 1 & 5.5297 & 5.5297 & 0.7768 & 0.529 \\
\hline & DENSITY & 1 & 16.4362 & 16.4362 & 2.3088 & 0.039 \\
\hline & POPULATION $\times$ DENSITY & 1 & 3.4896 & 3.4896 & 0.4902 & 0.804 \\
\hline & Residual & 116 & 825.7873 & 7.1189 & & \\
\hline
\end{tabular}
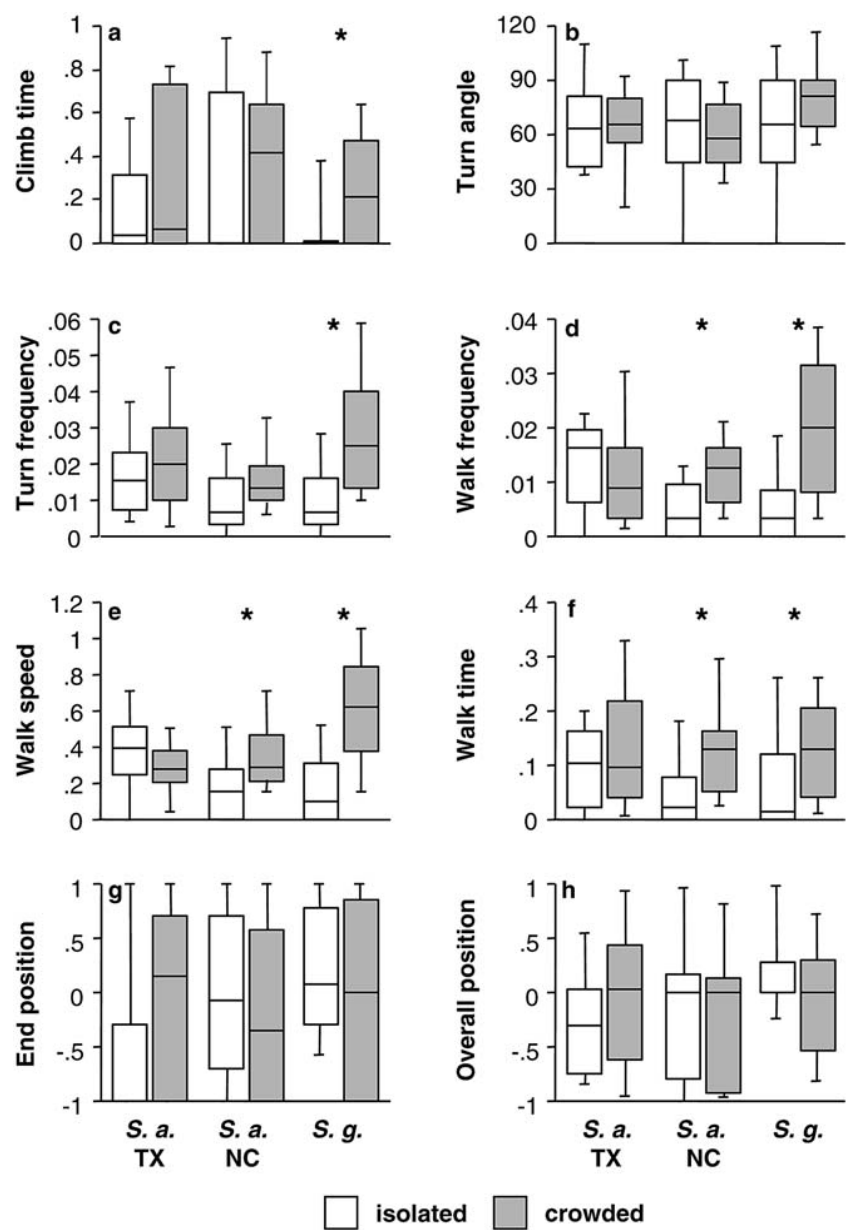

Fig. 1. Box plots depicting the effects of rearing density on six activity-related (a-f) and two position-related behaviors ( $g$ and $h$ ) in first instar S. americana (S.a.) from TX and NC populations as well as first instar S. gregaria (S.g.). Each box plot displays the median value with the ends of the boxes representing the 25 th and 75 th percentiles and the ends of the lines representing the 10th and 90th percentile values. Asterisks indicate significant comparisons between density treatments following sequential Bonferroni correction. The variables, End position and Overall position ( $\mathrm{g}$ and $\mathrm{h}$ ), can vary from -1 to 1 with positive values being closer to the stimulus group.
0.0001; associated $\alpha=0.0102$ ) (Fig. 1a, c). As was observed among crowded $S$. americana first instars (Mann-Whitney test results presented above), crowded $S$. gregaria first instars also exhibited significant densitydependent increases in Walk frequency $(U=652.5 ; P$ $<0.0001$; associated $\alpha=0.0073$ ), Walk speed ( $U=$ 537.5; $P<0.0001$; associated $\alpha=0.0064)$, and Walk time $(U=1002.50 ; P<0.0001$; associated $\alpha=0.0127)$ (Fig. 1d-f).

\subsubsection{Final instars}

The overall behavioral response of final instar nymphs to rearing density also differed between $S$. americana and $S$. gregaria as evidenced by the significant SPECIES $\times$ DENSITY interaction (Table 2). Examination of the underlying univariate behavioral differences revealed that the species were similar in that Climb time significantly increased among both crowded $S$. americana (Mann-Whitney test results presented above) and $S$. gregaria $(U=1086 ; P<0.0001$; associated $\alpha=$ 0.0064)(Fig. 2a). However, the species' behaviors differed because $S$. gregaria exhibited significant densitydependent increases, but $S$. americana did not, in the five remaining activity-related behaviors, Turn angle ( $U=671.0 ; P<0.0001$; associated $\alpha=0.0073)$, Turn frequency $(U=189.5 ; P<0.0001$; associated $\alpha=$ $0.0085)$, Walk frequency $(U=132.0 ; P<0.0001$; associated $\alpha=0.0102)$, Walk speed $(U=325.0 ; P<$ 0.0001 ; associated $\alpha=0.0127)$, and Walk time ( $U=$ 222.50; $P<0.0001$; associated $\alpha=0.017$ )(Fig. 2b-f).

\section{Discussion}

I have shown that $S$. americana retains variation for the expression of density-dependent behavioral changes, but its behavior can vary geographically between populations as well as within a population during development. The behavioral response of $S$. americana to changes in rearing density was reduced relative to that of $S$. gregaria in both the number of behaviors affected 

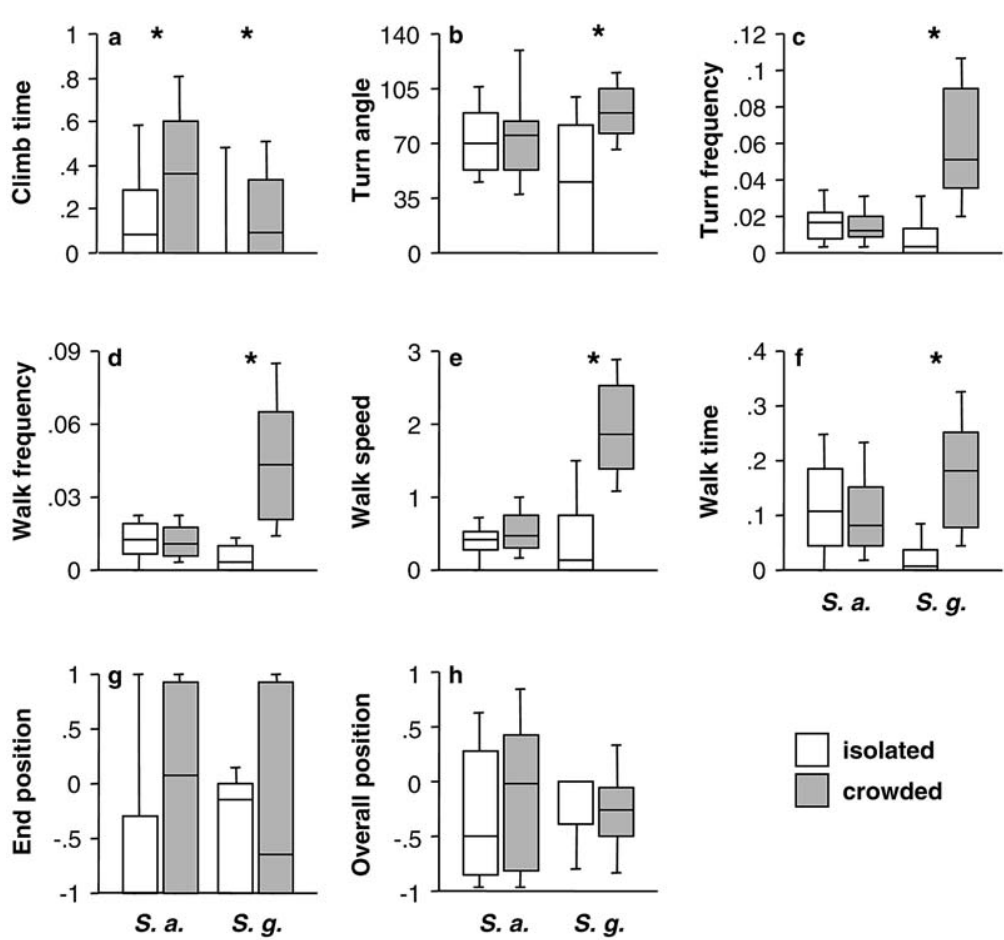

Fig. 2. Box plots depicting the effects of rearing density on six activity-related (a-f) and two position-related behaviors ( $g$ and $h$ ) in final instar S. americana (S.a.) (NC and TX populations combined) and S. gregaria (S.g.). Each box plot displays the median value with the ends of the boxes representing the 25th and 75th percentiles and the ends of the lines representing the 10th and 90th percentile values. Asterisks indicate significant comparisons between density treatments following sequential Bonferroni correction. The variables, End position and Overall position ( $\mathrm{g}$ and $\mathrm{h}$ ), can vary from -1 to 1 with positive values being closer to the stimulus group.

Table 2

Non-parametric MANOVA of the effects of species (SPECIES) and rearing density (DENSITY) on the overall behavior of first and final instar $S$. americana and $S$. gregaria. Due to a significant POPULATION x DENSITY interaction among first instar S. americana (Table 1), interspecific first instar comparisons were made with $S$. americana from only the NC population. Final instar comparisons include $S$. americana from both the TX and NC populations

\begin{tabular}{|c|c|c|c|c|c|c|}
\hline Instar & Source & $d f$ & SS & MS & $F$ & $P$ \\
\hline \multirow[t]{4}{*}{ First } & SPECIES & 1 & 25.0450 & 25.0450 & 4.4027 & 0.008 \\
\hline & DENSITY & 1 & 113.3812 & 113.3812 & 19.9316 & 0.001 \\
\hline & SPECIES $\times$ DENSITY & 1 & 20.0124 & 20.0124 & 3.5180 & 0.008 \\
\hline & Residual & 116 & 659.8687 & 5.6885 & & \\
\hline \multirow[t]{4}{*}{ Final } & SPECIES & 1 & 46.5649 & 46.5649 & 9.2647 & 0.001 \\
\hline & DENISTY & 1 & 223.8375 & 223.8375 & 44.5352 & 0.001 \\
\hline & SPECIES $\times$ DENSITY & 1 & 211.3074 & 211.3074 & 42.0422 & 0.001 \\
\hline & Residual & 236 & 1186.1542 & 5.0261 & & \\
\hline
\end{tabular}

and the relative magnitudes of density-dependent change.

\subsection{Geographic and developmental variation in $S$. americana}

First instar S. americana behavior varied between the $\mathrm{TX}$ and NC populations primarily due to significant density-dependent increases among the crowded NC insects in three activity-related behaviors, Walk frequency, Walk speed, and Walk time (Fig. 1). Although no sig- nificant density-dependent differences were detected among the TX first instars, their activity level was elevated independent of rearing density and was often more akin to the crowded as opposed to isolated NC first instars (Fig. 1c-f). The behavior of $S$. americana also varied with developmental stage within each of the populations. Only one activity-related behavior, Climb time, increased significantly in response to crowded rearing conditions among the final instars regardless of their population. This final instar response differed from the first instar responses in two ways. First, Climb time was 
not significantly affected by density in first instars from either the NC or TX population. Thus, crowded final instars from both populations expressed a new densitydependent behavior relative to the first instars. Second, significant increases in Walk frequency, Walk speed, and Walk time were no longer apparent among the final instar NC insects (Fig. 2d-f). This change appears to be due to an overall increase in activity levels among the isolated final instar NC insects (Figs. 1 and 2), and is similar to the elevated activity levels observed among the isolated first instar TX insects (Fig. 1).

\subsection{Comparison between $S$. americana and $S$. gregaria}

This study concurs with previous studies that have shown the behavioral response of $S$. gregaria to changes in rearing density to be relatively stable across developmental stages (Islam et al., 1994a, b; Simpson et al., 1999). The developmental changes in response to density observed here among $S$. americana contrasted with the relatively minor developmental changes observed in $S$. gregaria. In terms of the number of behaviors influenced by density, only one additional significant densitydependent increase in an activity-related behavior, Turn angle, occurred between the first and final instars of $S$. gregaria (Figs. 1 and 2). Due to the developmental changes in S. americana behavior observed here, the differences between $S$. americana and S. gregaria behavior became quite extreme by the final instar. Final instar $S$. americana exhibited fewer significant density-dependent changes than $S$. gregaria (1 of 8 vs. 6 of 8 ) and crowded $S$. gregaria tended to be much more active than either isolated or crowded S. americana (Fig. 2). Although final instar S. americana behavior was relatively unaffected by rearing density, the insects did not necessarily behave like isolated $S$. gregaria. It is interesting to note that the expression levels of all of the activity-related behaviors in final instar S. americana regardless of rearing density were greater than they were among isolated S. gregaria (Fig. 2a-f).

The possibility that minor differences in rearing conditions may have contributed to behavioral differences between the species cannot be ruled out. However, the conditions within each species were constant and suggest that the developmental differences in behavior observed between species in this study were not due to uncontrolled variables. Without detailed knowledge of the individual activity patterns of both species in the field, it is difficult to explain the significance of the observed behavioral differences between species as well as the variation observed within and between S. americana populations. Perhaps these differences reflect geographic and developmental variation in nymphal dispersal or foraging strategies. The possibility that parental effects influenced these observations is discussed below.

\subsection{Possibility of parental effects}

Maternal and paternal effects of crowding are known to influence the expression of phase changes across generations in locusts (Pener, 1991; Islam et al., 1994a, b; Simpson et al., 1999). The parental histories of the $S$. americana and $S$. gregaria nymphs observed in this study differed primarily in two ways. First, the rearing histories prior to capture of the adult $S$. americana from TX and NC populations were unknown, although both were low-density, non-outbreak populations at the time of collection. Thus, the possibility that the variation in behavior observed between $S$. americana populations in the first instar was due to uncontrolled differences in parental effects between populations cannot be ruled out. If this were the case, it implies that the TX population may have recently experienced high-density conditions, the effects of which were apparent in elevated activity levels of their isolated first instar progeny (Fig. 1). This seems unlikely, however, because I have collected $S$. americana adults at this site in previous years, including the year before, and their densities were always much less than 1 per $\mathrm{m}^{2}$.

The second difference in parental rearing history was that the crowded $S$. gregaria had been reared continuously under crowded conditions for a number of generations. This is less likely to be of importance in this study, however, because it is the loss of gregarious phase behaviors, rather than their gain, that is predominately influenced by transgenerational effects (Islam et al., 1994b). In other words, the effects of density on the behavior of crowded $S$. gregaria can occur very rapidly and are as extreme within a single generation as they are across generations. Assuming the same could be true in $S$. americana, this suggests that the difference between species in the magnitude of behavioral response to density is more likely due to genetic differences than epigenetic effects.

\subsection{Activity vs. attraction}

Gregarious phase behavior induced by high rearing density in S. gregaria is typified across developmental stages by an increase in both activity and mutual attraction (Islam et al., 1994a, b; Simpson et al., 1999). To varying degrees, both $S$. gregaria and $S$. americana exhibited significant density-dependent increases in activityrelated behaviors, but neither species exhibited any significant density-dependent changes in the two attractionrelated behaviors. Previous researchers using similar observational protocols have encountered variability in the detection of activity vs. attraction-related behavioral changes and speculated that this may be due to the arbitrary dimensions of the behavioral arenas (Islam et al., 1994b; Roessingh et al., 1998). Another possible explanation for the lack of a significant effect of rearing den- 
sity on attraction-related behaviors in this study could be the size of the stimulus groups employed in the behavioral assays. This study used stimulus groups of 10 insects, whereas other studies such as Islam et al. (1994a, b) and Roessingh et al. (1993) used larger stimulus groups of 50-100 first instar and 15 final instar nymphs, respectively. Olfactory stimuli as well as visual stimuli due to movement of stimulus group insects are likely to be greater from larger groups. Both movement and olfactory stimuli have been shown to influence the expression of phase-related behaviors in locust nymphs (Despland, 2001; Heifetz et al., 1996; Sword and Simpson, 2000).

A number of non-significant trends in this study suggest that attraction-related behaviors of both species varied in a locust-like manner in response to the presence of conspecifics in the arena. Among the first instar TX insects, for example, their overall position during the observation period was more or less unaffected by density and location of the stimulus group (Fig. 1h), but at the end of the assay, isolated insects tended to be farther from the stimulus group than their crowded counterparts (Fig. 1g). A similar trend was observed among final instars of both species. In S. americana, isolated final instars tended to be farther from the stimulus group than crowded insects at the end of the assay (Fig. 2 g). S. gregaria values for End position indicate that the isolated final instars were rarely near the stimulus group at the end of the assay, whereas the position of the crowded insects was more variable, suggesting that some crowded insects were less repelled by conspecifics (Fig. 2g).

\subsection{Evolution of differences in behavioral phase polyphenism}

Does $S$. americana rarely swarm because it lacks the genetic capacity to mount the appropriate density-dependent behavioral responses? Or is its lack of swarming simply because it exists in environments that are rarely conducive to swarm formation? There is a substantial amount of variation among different Schistoceca species in their expression of phase polyphenism (Kevan, 1943; Duck, 1944; Rowell and Cannis, 1971; Harvey, 1981; Sword, 1999). In some cases, such as the expression of density-dependent nymphal color change in S. emargin$a t a$, patterns of genetic variation for the expression plastic responses have been linked to variation in local ecological conditions (Sword, 2002; Dopman et al., 2002). Unfortunately, at this time we cannot determine if the reduced expression of nymphal behavioral phase polyphenism in $S$. americana relative to $S$. gregaria is related to its reduced tendency to swarm. Nor can we determine whether adaptive or neutral processes were responsible for the differences between species.

It is conceivable that extreme density-dependent behavioral changes have been maladaptive for S. amer- icana and as a result behavioral plasticity on par with that of $S$. gregaria has not been favored by natural selection. Alternatively, S. americana outbreaks are relatively rare and its populations may rarely reach densities high enough to induce the expression of density-dependent behavioral phase change. In such a case, we might suspect the relative lack of behavioral plasticity in S. americana to have evolved either (i) as a result of genetic drift in the absence of costs for the maintenance of plasticity (DeWitt et al., 1998), or (ii) due to selection against the costs of maintaining plasticity. If either of the two preceding scenarios is true, the expression of density-dependent behaviors by $S$. americana in the lab may be largely irrelevant in a current ecological context. In an evolutionary context, however, these behaviors could be important. Genetic variation for plastic responses in a trait can lurk as an unexpressed portion of the reaction norm and serve as an important source of variation for the expression of plastic traits when organisms encounter novel environments (Schlichting and Pigliucci, 1998).

\subsection{Future directions}

The reduced expression of behavioral phase polyphenism is associated with reduced tendency to swarm in $S$. americana, but a cause and effect relationship between these two phenomena cannot yet be established. The unique evolutionary history and biogeography of Schistocerca (Dirsh, 1974) has resulted in a large-scale natural experiment on the evolution of phase polyphenism and its relationship to swarming. A broader phylogenetic analysis of phase polyphenism that incorporates both swarming and non-swarming Schistocerca species will ultimately enhance our understanding of the relationship between the genetic and environmental factors that contribute to locust swarm formation. In the nearer term, the behavioral differences reported here suggest that $S$. americanca could serve as an important comparative tool for researchers as they unravel the genetic, physiological, and neurophysiological mechanisms underlying locust behavioral phase change.

\section{Acknowledgements}

Thanks to Marti Anderson for advice on using NPMANOVA and to Reg Chapman for commenting on the manuscript. Steve Simpson commented on an early draft of this manuscript and generously provided access to laboratory facilities and locusts at the University of Oxford. Marianne Feaver kindly collected grasshoppers in NC. Dan Hahn and Fabrizio Gabbiani helped wrangle grasshoppers in TX. Laura Senior assisted in rearing the grasshoppers and also read the manuscript. This project was funded in part by USDA-NRICGP grant \#9835302-6823. 


\section{References}

Anderson, M.J., 2000. NPMANOVA: a FORTRAN computer program for non-parametric multivariate analysis of variance (for any twofactor ANOVA design) using permutation tests. Department of Statistics, University of Auckland. http://www.stat.auckland.ac.nz/PEOPLE/marti/programs.htm

Anderson, M.J., 2001. A new method for non-parametric multivariate analysis of variance. Austral Ecology 26, 32-46.

Applebaum, S.W., Heifetz, Y., 1999. Density-dependent physiological phase in insects. Annual Review of Entomology 44, 317-341.

Bouaïchi, A., Simpson, S.J., Roessingh, P., 1996. The influence of environmental microstructure on the behavioural phase state and distribution of the desert locust Schistocerca gregaria. Physiological Entomology 21, 247-256.

Capinera, J.L., 1993. Host-plant selection by Schistocerca americana (Orthoptera: Acrididae). Environmental Entomology 22, 127-133.

Capinera, J.L., Scherer, C.W., Squitier, J.M., 2001. Grasshoppers of Florida. University Press of Florida, Gainesville.

Collett, M., Despland, E., Simpson, S.J., Krakauer, D.C., 1998. Spatial scales of desert locust gregarization. Proceedings of the National Academy of Sciences USA 95, 13052-13055.

Despland, E., 2001. Role of olfactory and visual cues in the attraction/repulsion responses to conspecifics by gregarious and solitarious desert locusts. Journal of Insect Behavior 14, 35-46.

Despland, E., Simpson, S.J., 2000a. The role of food distribution and nutritional quality in behavioural phase change in the desert locust. Animal Behaviour 59, 643-652.

Despland, E., Simpson, S.J., 2000b. Small-scale vegetation patterns in the parental environment influence the phase state of hatchlings of the desert locust. Physiological Entomology 25, 74-81.

Despland, E., Collett, M., Simpson, S.J., 2000. Small-scale processes in desert locust swarm formation: how vegetation patterns influence gregarization. Oikos 88, 652-662.

DeWitt, T.J., Sih, A., Wilson, D.S., 1998. Costs and limits of phenotypic plasticity. Trends in Ecology and Evolution 13, 77-80.

Dingle, H., 1996. Migration: The Biology of Life on the Move. Oxford University Press, Oxford.

Dirsh, V.M., 1974. Genus Schistocerca (Acridomorpha, Insecta). Dr. W. Junk B.V. Publishers, The Hague.

Dopman, E.B., Sword, G.A., Hillis, D., 2002. The importance of the ontogenetic niche in resource-associated diversification: evidence from a generalist grasshopper. Evolution 56, 731-740.

Duck, L.G., 1944. The bionomics of Schistocerca obscura (Fabr.). Journal of the Kansas Entomological Society 17, 105-119.

Fescemyer, H.W., 1993. Influence of phase polymorphism on the morphometric and physiological processes in preparation for insect migration. Journal of Agricultural Entomology 10, 219-237.

Harvey, A.W., 1979. Hybridization studies in the Schistocerca americana complex. I. The specific status of the Central American locust. Biological Journal of the Linnean Society 12, 349-355.

Harvey, A.W., 1981. A reclassification of the Schistocerca americana complex (Orthoptera: Acrididae). Acrida 10, 61-77.

Harvey, A.W., 1982. Hybridization studies in the Schistocerca americana complex. II. The Peruvian locust. Biological Journal of the Linnean Society 17, 217-223.

Harvey, A.W., 1983. Schistocerca piceifrons (Walker) (Orthoptera: Acrididae), the swarming locust of tropical America: a review. Bulletin of Entomological Research 73, 171-184.

Heifetz, Y., Voet, H., Applebaum, S.W., 1996. Factors affecting behavioral phase transition in the desert locust, Schistocerca gregaria (Forskal) (Orthoptera: Acrididae). Journal of Chemical Ecology 22, 1717-1734.

Islam, M.S., Roessingh, P., Simpson, S.J., McCaffery, A.R., 1994a. Effects of population density experienced by parents during mating and oviposition on the phase of hatchling desert locusts, Schistocerca gregaria. Proceedings of the Royal Society of London. Series B, Biological sciences 257, 93-98.

Islam, M.S., Roessingh, P., Simpson, S.J., McCaffery, A.R., 1994b. Parental effects on the behaviour and colouration of nymphs of the desert locust Schistocerca gregaria. Journal of Insect Physiology 40, 173-181.

Jago, N.D., Antoniou, A., Scott, P., 1979. Laboratory evidence showing the separate species status of Schistocerca gregaria, americana and cancellata (Acrididae, Crytacanthacridinae). Systematic Entomology 4, 133-142.

Kevan, D.K.McE., 1943. An account of Schistocerca flavofasciata (De Geer, 1773) in Trinidad. Bulletin of Entomological Research 34, 291-310.

Kuitert, L.C., Connin, R.V., 1952. Biology of the american grasshopper in the southeastern United States. Florida Entomologist 35, 22-33.

Pener, M.P., 1991. Locust phase polymorphism and its endocrine relations. Advances in Insect Physiology 23, 1-79.

Pener, M.P., Yerushalmi, Y., 1998. The physiology of locust phase polymorphism: an update. Journal of Insect Physiology 44, 365 377.

Roessingh, P., Simpson, S.J., James, S., 1993. Analysis of phaserelated changes in behaviour of desert locust nymphs. Proceedings of the Royal Society of London. Series B, Biological sciences 252, 43-49.

Roessingh, P., Bouaïchi, A., Simpson, S.J., 1998. Effects of sensory stimuli on the behavioural phase state of the desert locust, Schistocerca gregaria. Journal of Insect Physiology 44, 883-893.

Rowell, C.H.F., Cannis, T.L., 1971. Environmental factors affecting the green/brown polymorphism in the cyrtacanthacridine grasshopper Schistocerca vaga (Scudder). Acrida 1, 69-77.

Schlichting, C.D., Pigliucci, M., 1998. Phenotypic Evolution: A Reaction Norm Perspective. Sinauer Associates, Sunderland.

Simpson, S.J., McCaffery, A.R., Hägele, B.F., 1999. A behavioural analysis of phase change in the desert locust. Biological Reviews of the Cambridge Philosophical Society 74, 461-480.

Simpson, S.J., Despland, E., Hägele, B.F., Dodgson, T., 2001. Gregarious behavior in desert locusts is evoked by touching their back legs. Proceedings of the National Academy of Sciences USA 98, 3895-3897.

Sokal, R.R., Rohlf, F.J., 1995. Biometry, third ed. W.H. Freeman and Company, New York.

Sword, G.A., 1999. Density-dependent warning coloration. Nature 397, 217.

Sword, G.A., 2002. A role for phenotypic plasticity in the evolution of aposematism. Proceedings of the Royal Society of London. Series B, Biological sciences 269, 1639-1644.

Sword, G.A., Simpson, S.J., 2000. Is there an intraspecific role for density-dependent colour change in the desert locust? Animal Behaviour 59, 861-870.

Sword, G.A., Simpson, S.J., El Hadi, O.T.M., Wilps, H., 2000. Density-dependent aposematism in the desert locust. Proceedings of the Royal Society of London. Series B, Biological sciences 267, 63-68.

Uvarov, B.P., 1921. A revision of the genus Locusta (L.) (=Patchytylus Fieb.), with a new theory as to the periodicity and migrations of locusts. Bulletin of Entomological Research 12, 135-163.

Uvarov, B.P., 1966. Grasshoppers and Locusts Vol. 1. Cambridge University Press, Cambridge.

Uvarov, B.P., 1977. Grasshoppers and Locusts Vol. 2. Centre for Overseas Pest Research, London.

Wilson, K., Thomas, M.B., Blanford, S., Doggett, M., Simpson, S.J., Moore, S.L., 2002. Coping with crowds: density-dependent disease resistance in desert locusts. Proceedings of the National Academy of Sciences USA 99, 5471-5475. 